\title{
Knowledge, attitudes and practices of Canadian pediatric emergency physicians regarding short-term opioid use: a descriptive, cross-sectional survey
}

\author{
Megan Fowler MD, Samina Ali MDCM, Serge Gouin MDCM, Amy L. Drendel DO MS, \\ Naveen Poonai MD MSc, Maryna Yaskina PhD, Mithra Sivakumar RN, Esther Jun MD, \\ Kathryn Dong MD MSc; for Pediatric Emergency Research Canada
}

Abstract

Background: In the midst of the current opioid crisis, physicians are caught between balancing children's optimal pain management and the risks of opioid therapy. This study describes pediatric emergency physicians' practice patterns for prescribing, knowledge and attitudes regarding, and perceived barriers to and facilitators of short-term use of opioids.

Methods: We created a survey tool using published methodology guidelines and distributed it from October to December 2017 to all physicians in the Pediatric Emergency Research Canada database using Dillman's tailored design method for mixed-mode surveys. We performed bivariable binomial logistic regressions to ascertain the effects of clinically significant variables (e.g., training, age, sex, degree of worry regarding severe adverse events) on use of opioids as a first-line treatment for moderate pain in the emergency department, and prescription of opioids for moderate or severe pain for at-home use in children.

Results: Of the 224 physicians in the database, 136 (60.7\%) completed the survey $(60 / 111$ [54.1\%] women; median age 44 yr). Of the 136,74 $(54.4 \%)$ had subspecialty training. Intranasally administered fentanyl was the most commonly selected opioid for first-line treatment of moderate (47 respondents [34.6\%]) and severe (82 [60.3\%]) pain due to musculoskeletal injury. On a scale of 0 (not worried) to 100 (extremely worried), physicians' median score for worry regarding physical dependence was 6.0 (25th percentile 0.0 , 75th percentile 16.0), for worry regarding addiction 10.0 (25th percentile 2.0, 75th percentile 20.0) and for worry regarding diversion of opioids 24.5 (25th percentile 14.0, 75th percentile 52.0). On a scale of 0 (not at all) to 100 (extremely), the median score for influence of the opioid crisis on willingness to prescribe opioids was 22.0 (25th percentile 8.0, 75th percentile 49.0). The top 3 reported barriers to prescribing opioids were parental reluctance $(57[41.9 \%])$, lack of clear guidelines for pediatric opioid use $(35[25.7 \%])$ and concern about adverse effects (33 [24.3\%]). Binomial logistic regression did not identify any statistically significant variables affecting use of opioids in the emergency department or prescribed for use at home.

Interpretation: Emergency department physicians appeared minimally concerned about physical dependence, addiction risk and the current opioid crisis when prescribing opioids to children. Evidence-based development of guidelines and protocols for use of opioids in children may improve physicians' ability to manage pain in children responsibly and adequately.

$\mathrm{P}$ ain is one of the most common reasons for visits to the emergency department and accounts for up to $80 \%$ of visits in North America. ${ }^{1,2}$ However, inadequate analgesia for children remains a worldwide problem. ${ }^{3,4}$ The World Health Organization and the American Academy of Pediatrics have long recommended the addition of opioids for pain in children that does not respond to acetaminophen or nonsteroidal anti-inflammatory drugs or both. ${ }^{5,6} \mathrm{With}$ the current international opioid crisis and lack of clear national guidelines in Canada, physicians are caught between providing effective pain management and the risks of opioid therapy $^{7,8}$ This is especially relevant in the emergency department, which has been implicated in the current crisis., 90

There are limited studies describing physicians' knowledge, attitudes and barriers regarding prescribing opioids to children. A study from Brazil on pain management in pediat- ric inpatient wards showed that the most common barriers to pain management included fear of adverse drug reactions, fear of opioid dependence and tolerance, and lack of knowledge or understanding. ${ }^{11}$ In comparison, Ontario primary care physicians reported that they were most concerned about adult patients' running out of opioid medications early, demanding fit-in appointments, losing prescriptions and developing opioid addiction. ${ }^{12}$

\section{Competing interests: None declared.}

This article has been peer reviewed.

Correspondence to: Samina Ali, sali@ualberta.ca

CMAJ Open 2020. DOI:10.9778/cmajo.20190101 
The objectives of the present study were to describe pediatric emergency medicine physicians' patterns of prescribing short-term opioids in hypothetical scenarios of varying musculoskeletal injury pain in the emergency department and at discharge; their perceived knowledge and attitudes regarding prescribing short-term opioids, and their perceived barriers to and facilitators of prescribing short-term opioids; and to relate these findings to the physicians' demographic characteristics.

\section{Methods}

\section{Design, setting and participants}

This was a descriptive, cross-sectional survey of pediatric emergency physicians across Canada. Pediatric Emergency Research Canada is a network of health care providers interested in pediatric emergency medicine, with representation from all pediatric hospitals across the country. The 2017 Pediatric Emergency Research Canada physician database constituted our study population. This study was conducted at the Department of Pediatrics, University of Alberta between October and December 2017.

\section{Survey tool}

Four members of the research team (M.F., S.A., S.G. and E.J.) developed a survey tool following published guidelines for self-administered clinician surveys. ${ }^{13}$ An expert panel (representing pediatric emergency medicine, pain management, toxicology, addiction medicine and survey methodology) informed tool development. After item generation, item reduction and pretesting, the survey was pilot tested and reviewed for sensibility by 5 pediatric emergency physicians to ensure readability, and face and content validity (Appendix 1, available at www.cmajopen.ca/content/8/1/ E148/supp1/DC1).

The survey was available in English and French. The English-language survey was translated into French and then back-translated into English to ensure validity by the Pediatric Emergency Research Program at the Centre hospitalier universitaire Sainte-Justine, Montréal. The survey included 30 questions regarding physicians' demographic characteristics, knowledge and attitudes regarding potential risks and safe practice with opioid use (e.g., physical dependence, addiction), current practice, and perceived barriers to and facilitators of prescribing opioids (Appendix 2, available at www.cmajopen. $\mathrm{ca} /$ content/8/1/E148/suppl/DC1). Physicians were presented with 3 hypothetical musculoskeletal injury case scenarios to determine analgesic preference in the emergency department and at discharge. The cases represented mild (3/10), moderate $(6 / 10)$ or severe (9/10) pain, as defined by the World Health Organization's pain ladder. ${ }^{5}$ Participants were asked which medication(s) (in combination or as monotherapy) they would typically use for first- and second-line pain management in the emergency department, as well as recommendations for at-home pain management. They were also asked to select up to 3 opioids that they frequently prescribed or used for moderate to severe pain in children aged 6-23 months, 2-5 years, $6-12$ years and $13-18$ years.

\section{Survey implementation}

Participants were contacted from October to December 2017. We used Dillman's tailored design method for mixed-mode surveys to distribute the surveys. ${ }^{14}$ A prenotification email was sent on day 0 , followed by the electronic survey link on days 7 and 21. Consent was implied in completion of the survey. Based on pilot work, the time to complete the survey was 10-15 minutes. All email addresses were confirmed as active and current with Pediatric Emergency Research Canada.

Nonresponders to the electronic survey were mailed a paper survey on day 35. Study data were collected and managed with the use of the REDCap electronic data capture tool hosted by the Women and Children's Health Research Institute, Edmonton. ${ }^{15}$ Response validity and data checks were built into the REDCap database to avoid data entry errors, and participants were permitted to review and change their answers.

An arms-length research coordinator (M.S.) was responsible for distributing the survey, tracking participants' response status, mailing paper surveys, data entry and secure storage. The study team was blinded to the identity of the respondents and nonrespondents.

After completion of the survey, participants were offered a $\$ 5$ coffee gift card.

\section{Statistical analysis}

We summarized categorical data (e.g., sex, institution) with frequency distributions and reported continuous data (e.g., age, scales of worry and knowledge) using univariable summaries (means, medians, standard deviations, ranges). We performed bivariable binomial logistic regressions to ascertain the effects of clinically important variables (e.g., training, age, sex, degree of worry regarding severe adverse events) on use of opioids as a first-line treatment of moderate pain in the emergency department and prescription of opioids for moderate or severe pain for at-home use. A $p$ value of $<0.05$ was considered statistically significant. We performed all statistical analyses using SAS Version 9.4 (SAS Institute).

\section{Ethics approval}

This study received ethics approval from the University of Alberta's Health Research Ethics Board.

\section{Results}

At the time of study, the Pediatric Emergency Research Canada physician database included 224 physicians, including residents. Of the 224, $136(60.7 \%)$ completed the survey (113 electronically, 23 on paper). Respondents' demographic characteristics were representative of the Pediatric Emergency Research Canada physician database (Table 1). Participants were permitted to skip questions, and, therefore, response rates varied by question.

\section{Practice patterns for prescribing opioids and other analgesic agents}

Physicians' practice patterns for prescribing opioids for moderate to severe pain in the emergency department and at 


\begin{tabular}{|c|c|}
\hline Characteristic & $\begin{array}{l}\text { No. }(\%) \text { of } \\
\text { respondents† }\end{array}$ \\
\hline Female sex $(n=111)$ & $60(54.0)$ \\
\hline Age, yr, median (Q1, Q3) $(n=105)$ & $44(37,49)$ \\
\hline \multicolumn{2}{|l|}{ Province of practice $(n=111)$} \\
\hline British Columbia & $10(9.0)$ \\
\hline Alberta & $23(20.7)$ \\
\hline Saskatchewan & $2(1.8)$ \\
\hline Manitoba & $4(3.6)$ \\
\hline Ontario & $33(29.7)$ \\
\hline Quebec & $27(24.3)$ \\
\hline Nova Scotia & $7(6.3)$ \\
\hline Newfoundland and Labrador & $5(4.5)$ \\
\hline \multicolumn{2}{|l|}{ Training background $(n=117)$} \\
\hline Pediatric Emergency Fellowship & $74(63.2)$ \\
\hline FRCP/CCFP-EM Emergency Medicine & $19(16.2)$ \\
\hline General pediatrics training & $18(15.4)$ \\
\hline Currently in fellowship/training & $4(3.4)$ \\
\hline $\begin{array}{l}\text { Other (Family Medicine, Pediatric Critical } \\
\text { Care) }\end{array}$ & $2(1.7)$ \\
\hline $\begin{array}{l}\text { Years in clinical practice, median (Q1, Q3) } \\
(n=109)\end{array}$ & $19(5,19)$ \\
\hline $\begin{array}{l}\text { \% pediatric patients, median (Q1, Q3) } \\
(n=110)\end{array}$ & $100(100,100)$ \\
\hline $\begin{array}{l}\text { Personal history of medical opioid use } \\
(n=110)\end{array}$ & $54(49.1)$ \\
\hline Family history of medical opioid use $(n=110)$ & $65(59.1)$ \\
\hline \multicolumn{2}{|c|}{$\begin{array}{l}\text { Note: CCFP-EM = Canadian College of Family Physicians: Emergency Medicine, } \\
\text { FRCP = Fellow of the Royal College of Physicians, Q1 = 25th percentile, Q3 = } \\
75 \text { th percentile. } \\
\text { "Respondents were allowed to skip questions, and, therefore, response rates } \\
\text { vary. } \\
\text { †Except where noted otherwise. }\end{array}$} \\
\hline
\end{tabular}

discharge are presented in Table 2. The most commonly selected analgesics for use in the emergency department and for home management are presented in Figure 1. Ibuprofen was the most commonly selected analgesic for first-line emergency department management and home management for all 3 categories of pain. Intranasally administered fentanyl was the most commonly selected opioid for first-line treatment of moderate (47 respondents [34.6\%]) and severe (82 [60.3\%]) pain due to musculoskeletal injury in the emergency department.

Figure 2 depicts preferred opioids used in the emergency department and recommended at discharge, based on age. Morphine and fentanyl were the most commonly selected opioids in the emergency department, across all age groups; in the at-home setting, the most commonly selected opioid was morphine for oral use, followed by oxycodone and hydromorphone. Notably, over one-third of respondents (49 [36.0\%]) indicated that they would not prescribe opioids to be used at home to children less than 2 years of age.

\section{Knowledge regarding safe opioid use}

On a 100 -point scale where $0=$ extremely bad and $100=$ extremely good, physicians' median score for self-reported knowledge regarding safe opioid use was 73.0 (25th percentile $57.0,75$ th percentile 85.0 ) in the emergency department and 66.5 (25th percentile 48.0, 75 th percentile 78.0 ) for discharge recommendations.

Of the 127 respondents who answered the question concerning the best mode to deliver education for clinicians regarding safe opioid use in children, 49 (38.6\%) selected Webbased independent study, 29 (22.8\%) selected specific training session or course, 18 (14.2\%) selected conference or medical event, and $17(13.4 \%)$ selected video education sessions.

Sixty-two (48.1\%) of 129 respondents reported having an opioid-specific protocol or policy in their emergency department, and 14/129 (10.8\%) reported having a protocol for discharge advice. Ninety-one (74.0\%) of 123 physicians reported that having an opioid protocol in their emergency department would be helpful, specifically for morphine, fentanyl and hydromorphone.

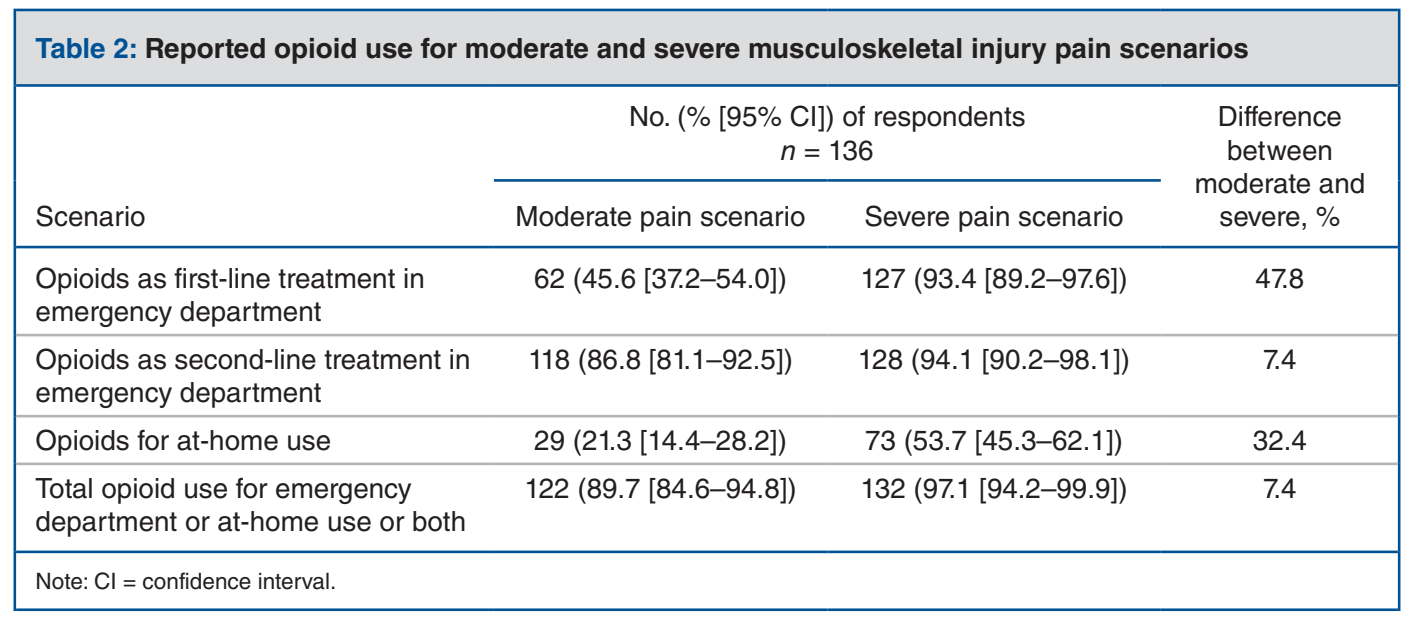


A

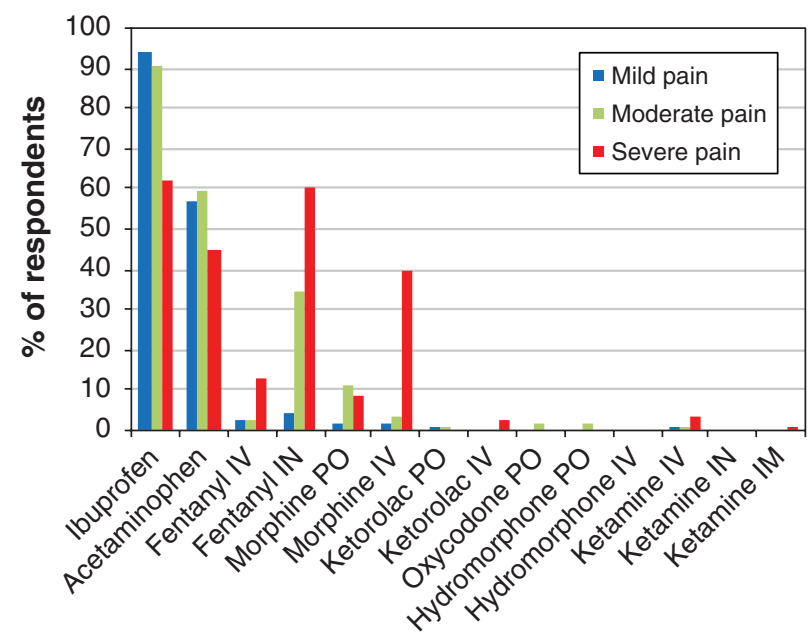

Analgesic agent

C

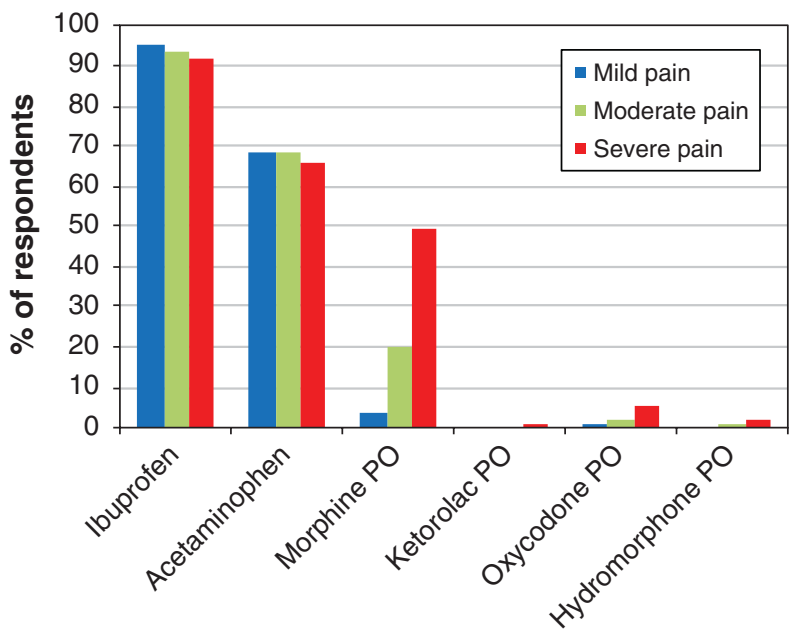

B

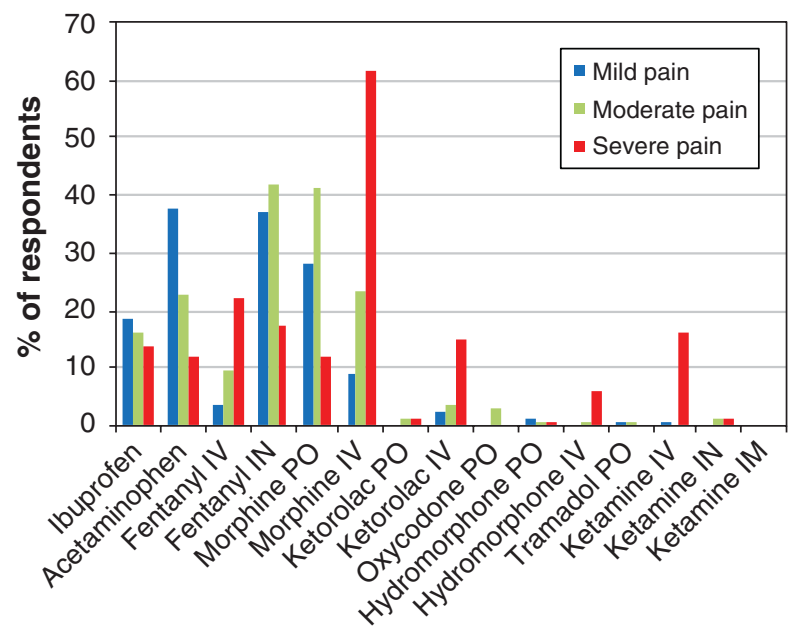

Analgesic agent

Analgesic agent

Figure 1: Physician-reported first-line pain medications in the emergency department $(A)$, second-line pain medications in the emergency department $(B)$ and pain medications at discharge (C) for mild (3/10), moderate (6/10) and severe (9/10) pain scenarios. $n=136$ for all questions. Note: IM = intramuscular, IN = intranasal, IV = intravenous, $\mathrm{PO}=$ per os (oral).

Physicians reported wanting more knowledge regarding the efficacy of various opioids (6/25 [24\%]), opioid dosing, indications, contraindications and duration of treatment $(6 / 25$ [24\%]), risk of opioid dependence, addiction and diversion $(4 / 25[16 \%])$, and opioid adverse effects $(3 / 25[12 \%])$.

\section{Attitudes regarding adverse effects}

On a 100-point scale where $0=$ not worried and $100=$ extremely worried, physicians' median score for worry regarding development of physical dependence in children was 6.0 (25th percentile $0.0,75$ th percentile 16.0 ), for worry regarding development of an opioid use disorder or addiction in children 10.0 (25th percentile 2.0, 75th percentile 20.0) and for worry regarding diversion of opioid prescriptions to others 24.5 (25th percentile 14.0, 75 th percentile 52.0). Physician's median scores for worry about severe adverse reactions at therapeutic dosages were as follows: apnea 16.5 (25th percentile $8.0,75$ th percentile 28.5 ) in the emergency department, 14.0 ( 25 th percentile $4.0,75$ th percentile 30.0 ) at home; hypotension 16.0 (25th percentile $7.0,75$ th percentile 28.0) in the emergency department, 9.0 (25 th percentile 2.0, 75 th percentile 19.0) at home; and cardiac arrest 4.0 (25th percentile $0.0,75$ th percentile 9.0$)$ in the emergency department, 4.0 (25 th percentile $0.0,75$ th percentile 12.0 ) at home.

On a 100 -point scale where $0=$ not at all and $100=$ extremely, the median score for influence of adverse effects (e.g., pruritis, nausea, vomiting, somnolence, constipation) on respondents' willingness to prescribe opioids to children was 
A

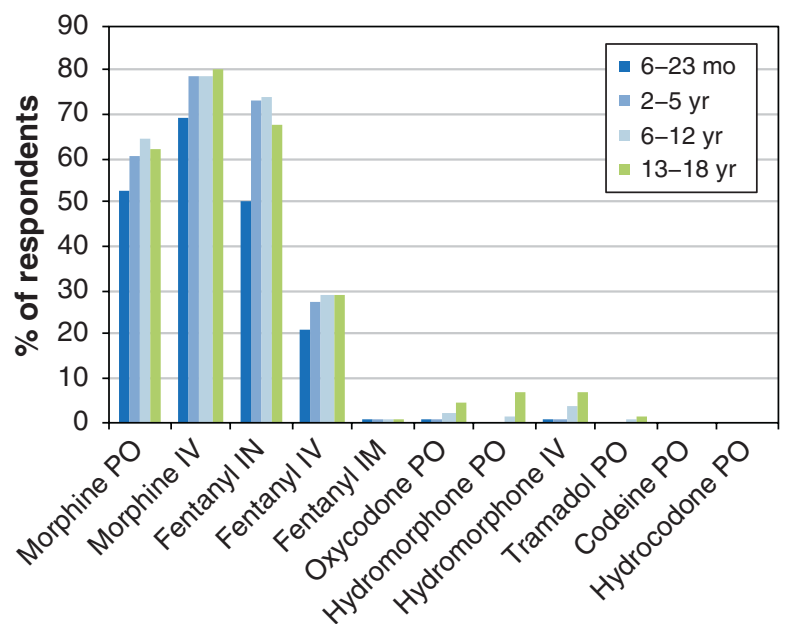

Opioid
B

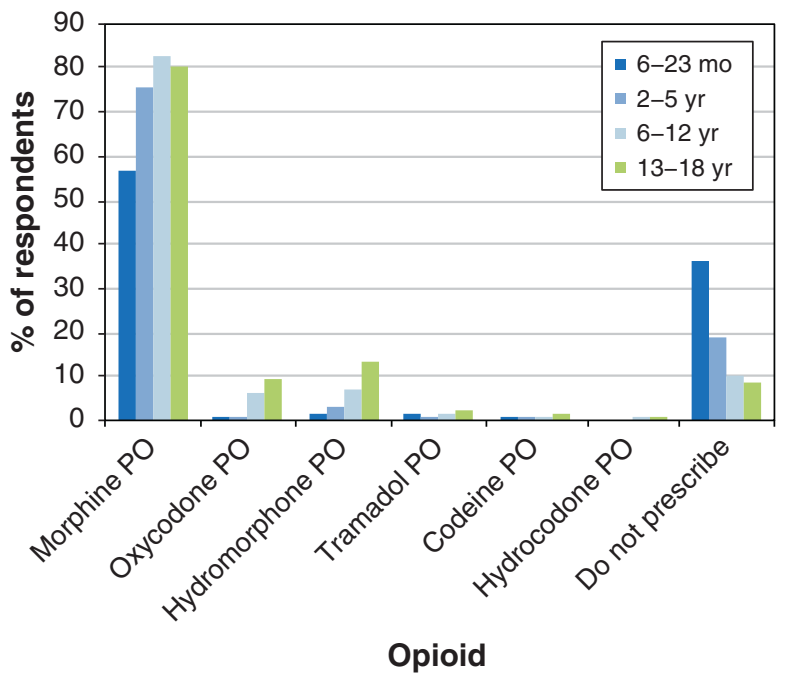

Figure 2: Most frequently reported opioids used in the emergency department $(A)$ and at discharge (B) for moderate or severe pain, according to patient age. $n=136$ for both questions. Note: IM = intramuscular, IN = intranasal, IV = intravenous, $\mathrm{PO}=$ per os (oral).

39.5 (25th percentile $16.0,75$ th percentile 61.5 ) and for influence of Canada's opioid crisis on respondents' willingness to prescribe opioids to children 22.0 (25th percentile 8.0, 75th percentile 49.0).

Table 3 presents physicians' self-reported frequency of counselling regarding safe opioid use. More than $80 \%$ of respondents $(105[81.4 \%])$ indicated that they often or always counselled families about the risks and adverse effects of opioids. In contrast, close to two-thirds (83 [64.3\%]) reported rarely or never performing a risk assessment before prescribing opioids, and less than $20 \%(21$ [16.3\%)] often or always counselled families about safe disposal of leftover medications.

\section{Perceived barriers to and facilitators of prescribing short-term opioids}

Table 4 presents perceived barriers to and facilitators of opioid use in the emergency department. Of the 52 respondents who answered the question regarding ways to remove barriers to responsible prescribing of opioids to children, 28 (54\%) reported the need for more education for physicians, nurses and caregivers, $8(15 \%)$ stated a need for research on safe opioid use in children, $7(13 \%)$ indicated a need for opioid guidelines, and $4(8 \%)$ mentioned the need for protocols.

\section{Additional analyses}

Binomial logistic regression to ascertain the effects of respondent demographic characteristics; worry regarding severe adverse reactions, opioid dependence, or opioid use disorders or addiction; and whether the respondent had an opioid protocol in his or her emergency department on the selection of opioids for first-line management of moderate pain in the emergency department and prescription of opioids for moderate or severe pain for at-home use did not identify any

\begin{tabular}{|c|c|c|c|}
\hline \multirow[b]{2}{*}{ Statement } & \multicolumn{3}{|c|}{$\begin{array}{l}\text { Response; no. (\%) of respondents } \\
\qquad n=129\end{array}$} \\
\hline & Often/always & Sometimes & Rarely/never \\
\hline $\begin{array}{l}\text { When deciding whether or not to prescribe opioids, I perform a } \\
\text { screening risk assessment (e.g., history of opioid use disorder) }\end{array}$ & $18(14.0)$ & $28(21.7)$ & $83(64.3)$ \\
\hline $\begin{array}{l}\text { When prescribing opioids, I counsel patients and parents about } \\
\text { potential risks and adverse effects of opioids }\end{array}$ & $105(81.4)$ & $14(10.8)$ & $10(7.8)$ \\
\hline $\begin{array}{l}\text { When prescribing opioids, I counsel patients and parents about } \\
\text { how to safely secure the medication away from the patient and } \\
\text { other family members }\end{array}$ & $53(41.1)$ & $30(23.3)$ & $46(35.7)$ \\
\hline $\begin{array}{l}\text { When prescribing opioids, I counsel patients and parents about } \\
\text { how to properly dispose of leftover medication }\end{array}$ & $21(16.3)$ & $17(13.2)$ & $91(70.5)$ \\
\hline
\end{tabular}


Table 4: Perceived barriers to and facilitators of opioid use in the emergency department

\begin{tabular}{|c|c|}
\hline Barrier/facilitator & $\begin{array}{l}\text { No. }(\%) \text { of } \\
\text { respondents* } \\
n=136\end{array}$ \\
\hline \multicolumn{2}{|l|}{ Barriers } \\
\hline Parental reluctance & $57(41.9)$ \\
\hline $\begin{array}{l}\text { Lack of clear guidelines for opioid use in } \\
\text { children }\end{array}$ & $35(25.7)$ \\
\hline Concern about adverse effects & $33(24.3)$ \\
\hline Concern about serious adverse events & $30(22.1)$ \\
\hline $\begin{array}{l}\text { Regulation of prescribing opioids (e.g., triplicate } \\
\text { prescription needed) }\end{array}$ & $29(21.3)$ \\
\hline Assessing pain in younger children is difficult & $27(19.8)$ \\
\hline Lack of comfort with medication dosing & $21(15.4)$ \\
\hline $\begin{array}{l}\text { Policy regarding nursing/monitoring of patients } \\
\text { who receive opioids }\end{array}$ & $21(15.4)$ \\
\hline Concern for opioid use disorders and addiction & $18(13.2)$ \\
\hline Lack of adequate research to guide practice & $17(12.5)$ \\
\hline Consultant preference & $16(11.8)$ \\
\hline Concern for opioid dependence & $13(9.6)$ \\
\hline $\begin{array}{l}\text { Fear that opioids can mask symptoms and lead } \\
\text { to missed diagnoses }\end{array}$ & $3(2.2)$ \\
\hline Other & $5(3.7)$ \\
\hline \multicolumn{2}{|l|}{ Facilitators } \\
\hline $\begin{array}{l}\text { Personal clinical experience with opioid use in } \\
\text { children }\end{array}$ & $108(79.4)$ \\
\hline $\begin{array}{l}\text { Opportunity to monitor patients in emergency } \\
\text { department }\end{array}$ & $90(66.2)$ \\
\hline $\begin{array}{l}\text { Parental approval and understanding of } \\
\text { benefits/adverse effects of opioids }\end{array}$ & $60(44.1)$ \\
\hline $\begin{array}{l}\text { Good research available on opioid use in } \\
\text { children }\end{array}$ & $51(37.5)$ \\
\hline Documented pain scores & $51(37.5)$ \\
\hline $\begin{array}{l}\text { Opioid-specific protocols in institution/ } \\
\text { emergency department }\end{array}$ & $47(34.6)$ \\
\hline $\begin{array}{l}\text { Patient known to be not opioid naive and has } \\
\text { used opioids safely in the past }\end{array}$ & $35(25.7)$ \\
\hline Other & $2(1.5)$ \\
\hline
\end{tabular}

statistically significant variables (Appendix 3, available at www.cmajopen.ca/content/8/1/E148/supp1/DC1).

\section{Interpretation}

Our participants reported high ibuprofen use for management of pain in children. Opioids were most commonly selected for second-line management of moderate and severe pain in the emergency department. The physicians in our study appeared to be minimally concerned about opioid dependence, addiction risk and the opioid crisis when prescribing opioids to children.

Intranasally administered fentanyl was the opioid most commonly selected by emergency physicians for first-line treatment of moderate and severe pain in children. The intranasal route has become a popular route of delivering medications, as they can be administered quickly and without intravenous insertion. ${ }^{16}$ It is interesting to note that the reported intranasal fentanyl use was high $(60 \%$ for first-line management of severe pain) given the lack of definitive evidence regarding its efficacy. The authors of a 2014 Cochrane review evaluating intranasal fentanyl therapy were unable to draw any conclusions comparing its efficacy to parenteral morphine therapy..$^{16}$ Subsequent modest-sized trials of intranasal fentanyl therapy have shown it to be comparable to intranasal ketamine therapy for suspected fractures. ${ }^{17,18}$ However, large multicentre trials are urgently needed to determine the efficacy of intranasal fentanyl therapy definitively.

The selection of opioids for second-line management of moderate and severe pain in the emergency department is congruent with international recommendations. ${ }^{5,6}$ The high ibuprofen use reported is in line with the current evidence on safety and efficacy for managing acute pain from musculoskeletal injury in children. ${ }^{19}$

The risk of opioid addiction and misuse in children is not clearly understood, and there is conflicting evidence from recent studies. ${ }^{20,21}$ McCabe and colleagues ${ }^{21}$ found that legitimate use of prescription opioids in adolescence was not associated with the development of a substance use disorder; however, a history of nonmedical use of prescription opioids in adolescence was associated with increased odds of substance use disorder. Miech and colleagues ${ }^{20}$ reported that prescribing opioids to adolescents was independently associated with a $33 \%$ increase in risk of future opioid misuse. Interestingly, Miech and colleagues ${ }^{20}$ also reported no associated risk for future opioid misuse among adolescents with reported use of alcohol and illegal drugs. With the current lack of strong guiding evidence regarding risk of future misuse after medical use of opioids in childhood, it is difficult to conclude how worried physicians "should" be about this risk.

Until recently, codeine was the most commonly prescribed orally administered opioid for children. ${ }^{22}$ In 2013, Health Canada advised no codeine use in children less than 12 years; ${ }^{23}$ this advisory challenged physicians to change their practice patterns and search for an alternative opioid for oral use. There have been 3 recent trials studying oral morphine therapy for musculoskeletal injury. ${ }^{24-26}$ In 2 trials, the investigators compared oral morphine therapy to ibuprofen, alone and in combination, and found no significant difference in efficacy. ${ }^{24,25}$ In addition, all 3 trials showed significantly more adverse effects in the morphine group than in the ibuprofen group. In contrast, physicians in our study reported that orally administered morphine was their preferred opioid for moderate and severe pain at discharge.

Physicians indicated they want more research, education and opioid-specific protocols and guidelines for pain management in children. After parental reluctance, lack of clear guidelines 
and concern for adverse effects were the top reported barriers to prescribing opioids in the emergency department.

Over the past 10 years, there has been a $53 \%$ increase in hospital admissions for opioid poisonings in Canada. ${ }^{27}$ Youth aged 15-24 years have the fastest growing rates of hospital admissions, and intentional poisonings were the most prevalent for this age group, accounting for $44 \%$ of these admissions. ${ }^{27}$ These numbers are alarming, and, in response to the opioid crisis, the Government of Canada developed a Joint Statement of Action to Address the Opioid Crisis. ${ }^{28,29}$ In the United States, the Centers for Disease Control and Prevention produced the 2016 Guidelines for Prescribing Opioids for Chronic Pain..$^{30}$ These documents fail to address the pediatric population, and there is worry that they are being extrapolated inappropriately to children. ${ }^{31}$ The current World Health Organization guidelines target persisting or chronic pain rather than acute pain, ${ }^{5}$ and the American Academy of Pediatrics guideline, although still helpful, predates the opioid crisis and does not provide direct guidance regarding choice of medications, dosing or length of therapy. ${ }^{6}$

Health care leaders and policy-makers must examine the current evidence and, with the help of experts in pediatric pain management, develop national guidelines in Canada regarding the indication for opioids, recommended dosing, duration of treatment and safety concerns for opioid use in children. ${ }^{31}$ Until then, Canadian physicians are left without clear guidance on how to prescribe opioids to children responsibly and adequately. Rigorous trials examining the effectiveness and safety of opioids in children are lacking, however.

Few physicians reported performing a screening risk assessment before prescribing opioids or counselling families about how to dispose of leftover medication properly. Clinicians need a quick, reliable, validated screening tool for identifying children at higher risk for complications related to opioid use in the emergency department. Furthermore, knowing that most people with nonmedical use of prescription opioids obtain opioids from family members or friends, ${ }^{32,33}$ physicians must counsel patients about proper disposal of unused medications.

Future robust studies examining the risk of opioid use disorder in children receiving short-term opioids may help guide clinical decision-making. Qualitative studies examining physicians' decision-making steps would help us understand physicians' barriers to and facilitators of prescribing opioids to children.

\section{Limitations}

Our study has several limitations. We had a modest response rate of $60.7 \%$; a higher response rate may have led to more generalizable results. We created a survey tool, which has not been validated. As with any survey study, the answers provided may have been influenced by respondent and recall bias. The Pediatric Emergency Research Canada database includes physicians who practise primarily at pediatric tertiary care centres; therefore, our results may not be generalizable to community physicians. Since we used hypothetical scenarios, our results cannot be generalized automatically to real-life scenarios. Finally, our scenarios did not explicitly provide information regarding mental health status or personal or family history of opioid use disorder or addiction, which may have influenced physicians' decisions in choosing opioids for pain management.

\section{Conclusion}

Intranasally administered fentanyl was the most commonly selected opioid for first-line management of moderate and severe pain in children in the emergency department, and opioids were most commonly selected for second-line management of moderate and severe pain. Physicians appear to be minimally concerned regarding opioid dependence, addiction risk and the current opioid crisis when prescribing opioids to children. There is an urgent need for robust evaluation of opioid dependence and addiction risk in children receiving opioids to inform physicians' patient-counselling practices. Development of opioid-specific guidelines and protocols would likely improve physicians' ability to manage pain for children responsibly and adequately.

\section{References}

1. Ali S, Drendel AL, Kircher J, et al. Pain management of musculoskeletal injuries in children: current state and future directions. Pediatr Emerg Care 2010; 26:518-24, quiz 525-8

2. Todd KH, Ducharme J, Choiniere M, et al.; PEMI Study Group. Pain in the emergency department: results of the pain and emergency medicine initiative (PEMI) multicenter study. 7 Pain 2007;8:460-6.

3. Cimpello LB, Khine H, Avner JR. Practice patterns of pediatric versus general emergency physicians for pain management of fractures in pediatric patients. Pediatr Emerg Care 2004;20:228-32.

4. Drendel AL, Brousseau DC, Gorelick MH. Pain assessment for pediatric patients in the emergency department. Pediatrics 2006;117:1511-8.

5. WHO guidelines on the pharmacological treatment of persisting pain in children with medical illnesses. Geneva: World Health Organization; 2012.

6. American Academy of Pediatrics. Committee on Psychosocial Aspects of Child and Family Health; Task Force on Pain in Infants, Children, and Adolescents. The assessment and management of acute pain in infants, children, and adolescents. Pediatrics 2001;108:793-7.

7. Schechter NL. Pediatric pain management and opioids: the baby and the bathwater. 7AMA Pediatr 2014;168:987-8.

8. Cheng T, DeBeck K. Between a rock and a hard place: prescription opioid restrictions in the time of fentanyl and other street drug adulterants. Can $\mathcal{F}$ Public Health 2017;108:e325-7.

9. Barnett ML, Olenski AR, Jena AB. Opioid-prescribing patterns of emergency physicians and risk of long-term use. N Engl F Med 2017;376:663-73.

10. Upadhye S. Creating opioid dependence in the emergency department. CFEM 2018;20:100-3.

11. de Freitas GR, de Castro CG Jr, Castro SM, et al. Degree of knowledge of health care professionals about pain management and use of opioids in pediatrics. Pain Med 2014;15:807-19.

12. Wenghofer EF, Wilson L, Kahan M, et al. Survey of Ontario primary care physicians' experiences with opioid prescribing. Can Fam Physician 2011;57:324-32.

13. Burns KE, Duffett M, Kho ME, et al.; ACCADEMY Group. A guide for the design and conduct of self-administered surveys of clinicians. CMA7 2008;179: 245-52.

14. Dillman DA, Smyth JD, Christian LM. Internet, mail, and mixed-mode surveys: the tailored design method. 3rd ed. Hoboken (NJ): John Wiley \& Sons; 2009.

15. Harris PA, Taylor R, Thielke R, et al. Research electronic data capture (REDCap) - a metadata driven methodology and workflow process for providing translational research informatics support. 7 Biomed Inform 2009; 42:377-81.

16. Murphy A, O'Sullivan R, Wakai A, et al. Intranasal fentanyl for the management of acute pain in children. Cochrane Database Syst Rev 2014;(10): CD009942.

17. Graudins A, Meek R, Egerton-Warburton D, et al. The PICHFORK (Pain in Children Fentanyl or Ketamine) trial: a randomized controlled trial comparing intranasal ketamine and fentanyl for the relief of moderate to severe pain in children with limb injuries. Ann Emerg Med 2015;65:248-54.e1.

18. Frey TM, Florin TA, Caruso $M$, et al. Effect of intranasal ketamine vs fentanyl on pain reduction for extremity injuries in children: the PRIME randomized clinical trial. FAMA Pediatr 2019;173:140-6. 
19. Le May S, Ali S, Khadra C, et al. Pain management of pediatric musculoskeletal injury in the emergency department: a systematic review. Pain Res Manag 2016;2016:4809394.

20. Miech R, Johnston L, O'Malley PM, et al. Prescription opioids in adolescence and future opioid misuse. Pediatrics 2015;136:e1169-77.

21. McCabe SE, Veliz P, Schulenberg JE. Adolescent context of exposure to prescription opioids and substance use disorder symptoms at age 35: a national longitudinal study. Pain 2016;157:2173-8.

22. Kircher J, Drendel AL, Newton AS, et al. Acute pediatric musculoskeletal pain management in North America: a practice variation survey. Clin Pediatr (Phila) 2014;53:1326-35

23. Health Canada's review recommends codeine only be used in patients aged 12 and over. Ottawa: Health Canada; modified 2016 July 28. Available: http://healthycanadians.gc.ca/recall-alert-rappel-avis/hc-sc/2013/33915a-eng. php (accessed 2019 Dec. 29).

24. Poonai N, Bhullar G, Lin K, et al. Oral administration of morphine versus ibuprofen to manage postfracture pain in children: a randomized trial. CMAf 2014:186:1358-63.

25. Poonai N, Datoo N, Ali S, et al. Oral morphine versus ibuprofen administered at home for postoperative orthopedic pain in children: a randomized controlled trial. CMA7 2017;189:E1252-8.

26. Le May S, Ali S, Plint AC, et al.; Pediatric Emergency Research Canada (PERC). Oral analgesics utilization for children with musculoskeletal injury (OUCH trial): an RCT. Pediatrics 2017;140:e20170186.

27. Opioid-related harms in Canada. Ottawa: Canadian Institute for Health Information; 2017. Available: www.longwoods.com/articles/images/opioid-harms -chart-book-en.pdf (accessed 2020 Jan. 22).

28. Foint statement of action to address the opioid crisis: a collective response (annual report 2016-2017). Ottawa: Canadian Centre on Substance Abuse and Addiction; 2017.

29. Busse JW, Craigie S, Juurlink DN, et al. Guideline for opioid therapy and chronic noncancer pain. CMA7 2017;189:E659-66.

30. Centers for Disease Control and Prevention Public Health Service US Department of Health and Human Services. Guideline for prescribing opioids for chronic pain. 7 Pain Palliat Care Pharmacother 2016;30:138-40.

31. Schechter NL, Walco GA. The potential impact on children of the CDC guideline for prescribing opioids for chronic pain: above all, do no harm. fAMA Pediatr 2016;170:425-6.

32. Jones CM, Paulozzi LJ, Mack KA. Sources of prescription opioid pain relievers by frequency of past-year nonmedical use: United States, 2008-2011. 7AMA Intern Med 2014;174:802-3.

33. Boyd CJ, McCabe SE, Cranford JA, et al. Prescription drug abuse and diversion among adolescents in a southeast Michigan school district. Arch Pediatr Adolesc Med 2007;161:276-81.
Affiliations: Department of Pediatrics (Fowler, Ali, Sivakumar, Jun) and Women and Children's Health Research Institute (Ali, Yaskina), University of Alberta, Edmonton, Alta.; Department of Pediatric Emergency Medicine (Gouin), Centre hospitalier universitaire Sainte-Justine, Montréal, Que.; Section of Emergency Medicine (Drendel), Department of Pediatrics, Children's Hospital of Wisconsin and Medical College of Wisconsin, Milwaukee, Wisc.; Division of Emergency Medicine (Poonai), London Health Sciences Centre, Western University, London, Ont.; Department of Emergency Medicine (Dong), University of Alberta, Edmonton, Alta.

Contributors: Samina Ali conceived the study. Megan Fowler and Samina Ali designed the study. Mithra Sivakumar coordinated the study and acquired the data. Megan Fowler and Maryna Yaskina analyzed the data. Samina Ali interpreted the data. Amy Drendel contributed as an expert in pediatric pain management. Kathryn Dong contributed as an expert in addiction medicine. Megan Fowler drafted the manuscript. Samina Ali, Serge Gouin, Amy Drendel, Naveen Poonai, Maryna Yaskina, Mithra Sivakumar, Esther Jun and Kathryn Dong revised the manuscript critically for important intellectual content. All of the authors approved the final version to be published and agreed to be accountable for all aspects of the work.

Funding: This study was supported by the Women and Children's Health Research Institute Resident Grant to Megan Fowler and Samina Ali (2017-2018).

Acknowledgements: The authors thank Pediatric Emergency Research Canada for their support and access to their physician database. They acknowledge Manasi Rajagopal for project coordination and administrative support. They thank their expert panel for help with survey tool development: Sylvie Le May, Dr. Garth Meckler, Dr. Sarah Curtis, Dr. Shazma Mithani and Dr. Michael Reider. They also thank the physicians who helped with pilot and clinical sensibility testing, and all those who participated in the survey.

Supplemental information: For reviewer comments and the original submission of this manuscript, please see www.cmajopen.ca/content/8/1/ E148/suppl/DC1. 